Z. PERJÉs

INTRODUCTION TO TWISTOR PARTICLE THEORY

Hungarian Fcademy of Sciences CENTRAL RESEARCH INSTITUTE FOR PHYSICS 


\section{INTRODUCTION TO TWISTOR PARTICLE THEORY}

zoltán Perjés

Central Research Institute for Physics

H-1525 Budapest 114, P.O.B. 49, Hungary 


\section{ABSTRACT}

Twistor theory of Penrose is a framework for describir.g quantized matter and geometry. An introduction is given into the theory hoping to encourage applications in particle physics. Interrelations among causality, conformal invariance and spinors are discussed. The structures representing. twistors in space-time are considered, followed by a description of zero-mass free fields using holomorphic twistor functions. Some relevant notions of cohomology theory are summarized. An intermediate-stage approach to the inclusion of rest-mass in twistor theory is worked out.

\section{АННОТАЦИЯ}

Теория твисторов Пенроэа является основой для описания квантованных частиц и геометрии. Дается введение в теорию для стимуляции ее применения в области физики элементарных частиц. Обсуждается взаимосвязь между причинностью, конформной инвариантностью и спинорами. Изучаются структуры, представляющие твисторы в пространстве-времени, и описываются безмассовые поля с применением голоморфных твисторных функцин. Даются некоторые соответствующие реэультаты теории когомологии. Дается твисторныи метод описания массы покоя.

\section{KIVONAT}

A Penrose-féle tvistorelmélet kvantumos részecskék és geometria leirását célozza. Részecskefizikai alkalmazásait elôsegitendõ, bevezetést nyujtunk az elméletbe. Tárgyaljuk az okság, konformis invariancia és a spinorok kapcsolatát. Foglalkozunk tvisztorok ábrázolásával a téridôben, majd a zérus tömegü terek leirásával analitikus tvisztor függvények segitségével. Ismertetjủk a kohomológiaielmélet néhány felhasználandó eredményét. Tvisztoreljárást adunk meg a nyugalmi tömeg tárgyalására. 


\section{BASIC PHILOSOPHY}

There have been instances in the past of particle physics when the validity of some fundamental law of nature was victoriusly reaffirmed and speculations doubting that law were put aside. Remember the apparent non-conservation of momentum in the $\beta$-decay, a paradox resolved by the discovery of neutrino. Then the rigorous formulation of quantum field theory owes much to our insistence on the validity of a variational principle.

Such a tendency should not be all that surprizing; as physics probes more deeply into the fundamentals, our understanding is guided by few surviving principles. Many of us believe, for example, that some form of causality must be among the ultimate laws of particle theory. Causality has become a basic ingredient of analytic S-matrix theory, considered once the avenue to particles. I think that ambitious analyticity programmes did not fail for technical reasons. I blame S-matrix theory on not formulating clearly the precise notion of causality to be connected with analyticity [1]. In particular, our description of fundamental particles must be intrinsically relativistic. Well, the relativistic version of causality is a study of light cones [2] rather than of any form of simultaneity. And this light-cone structure of space-time is a primary object of twistor theory, a framework devised by Penrose for describing quantized matter and geometry.

The case for the quantum nature of empty space geometry has not always been compelling. Today, Professor Symanzik told us in fine detalls about the Casimir effect or the pressure of quantum fluctuations of empty space [3]. This effect now is an experimental fact.

Twistor theory transcends Einstein's relativity which it contains as a classical limit. Space-time geometry occupies a dual status in general relativity: it is both a background and the physical field of gravitation. I shall now briefly describe the architecture of space-time as pictured in general relativity [4], which will be subject to the process of quantization.

Initially we have a set of events $p, q, r, \ldots$ such as, say, the collision of two particles. There is a notion of continuity in measurements which imposes a topology on this set. We next introduce co-ordinates by mapping onto open sets of the four-dimensional Euclidean.space $\mathbb{R}^{4}$ and requiring 


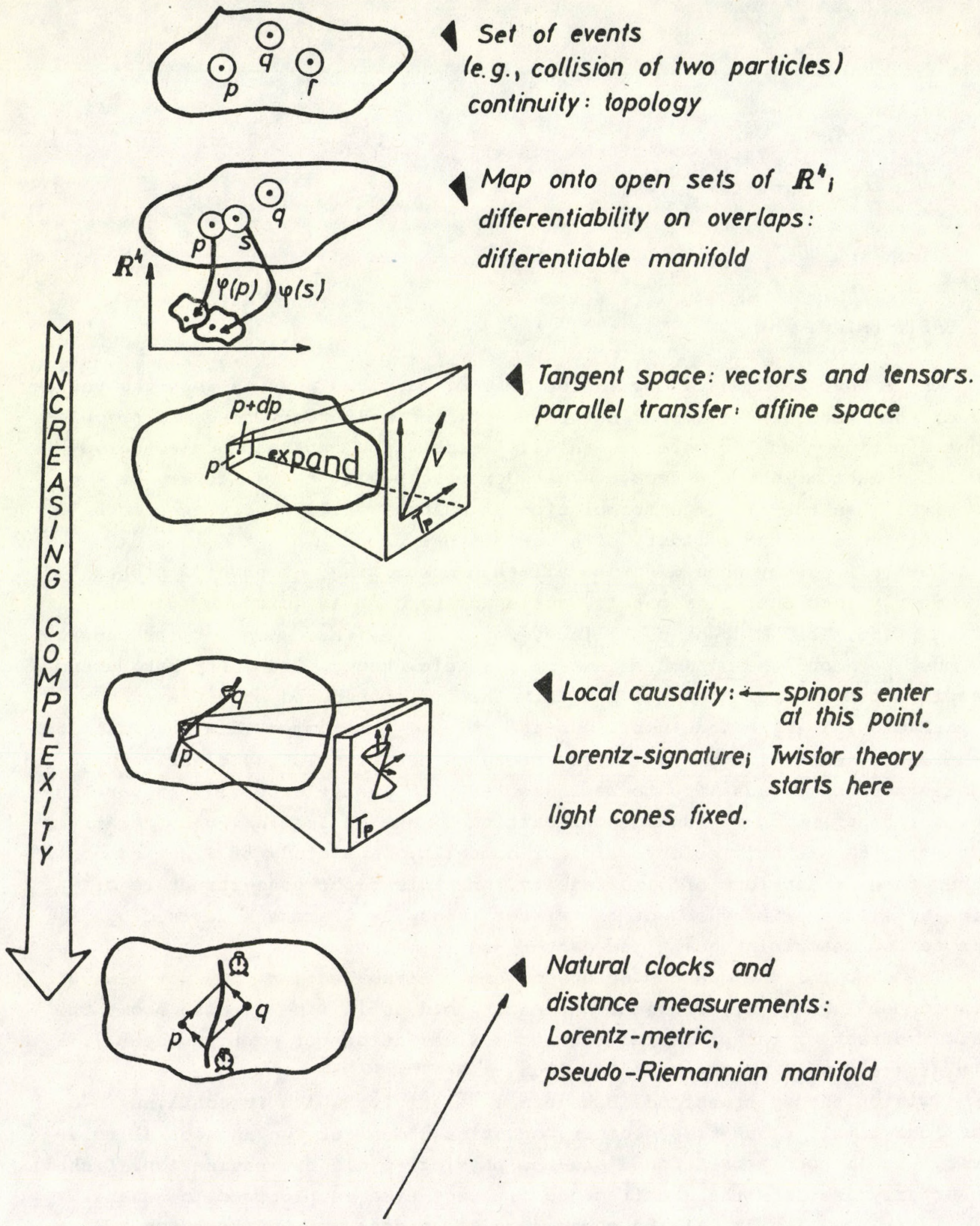

Many attempts at quantisation start here

Fig. 1. The architecture of space-time as described by general relativity 
differentiability on overlaps of the open sets. We have then converted our space into a differentiable manifold. The powerful machinery of differential geometry is thus at our disposal: we can consider linear spaces of vectors and tensors (such as the tangent space) attached to points of the manifold and we can define parallel transfer of the these spaces (Fig. 1).

Given a differentiable manifold, it is possible to endow 1 t with a positive definite metric [5]. However, this is not what Nature suggests to us to do next. Local causality, expressing the nonexistence of faster-than-light particles, fixes a Lorentz-signature in tangent spaces. Thus we divide tangent space into the orbits of time-like, null and space-like vectors. On top of the hierarchy of structures we have a Lorentzian metric which is measurable by using clocks and light signals. This makes a pseudo-Riemannian manifold.

The imposition of a Lorentz-signature means effectively that we have the length of vectors up to a positive constant factor, and amounts to fixing the light cones. The structures on null cones are suitably studied by using spinors, as will be shown in section 2. (It would be mistaken to think, however, that twistor theory has the aim to extend the utility of spinors to curved space. The case is rather that the use of spinors proves convenient). A space-time point is uniquely characterized by its own light cone and any light cone is uniquely characterized by all its null generators. A null generator represents a null twistor in space-time (Cf. Sec. 3).

Twistors form a 4 dimensional complex linear space $\mathbb{T}$. In addition to its intimate relation with the light-cone structure of space-time, twistor space possesses some features of a Hilbert space of quantum states. How is it possible for the apparently unrelated notions of a geometrical space and of the complex space of probability amplitudes to merge? This has been illustrated by Penrose [6] on the simple case of a spin-1/2 particle.

Ignoring degrees of freedom other than spin, a state $\mid a>$ of the particle can be characterized by the direction of the "good" quantization axis. By this I mean the direction of the axis which gives the value $1 / 2$ for the spin projection quantum number. Clearly, the set of possible directions in 3-space yields the two-sphere $\mathbb{S}^{2}$. Thus the Hilbert space of states is related to ordinary space. The relation can be made more explicit by stereographic projection (Fig. 2) onto the complex plane. An arbitrary (but normalized) state is a linear superposition of the $\operatorname{spin-up}(\zeta=\infty)$ and $\operatorname{spin-down}(\zeta=0)$ states :

$$
|a\rangle=\xi^{0}|+\rangle+\xi^{1}|\uparrow\rangle
$$

Dropping the over-all quantum mechanical phase factor, the state $\mid$ a $\rangle$ is uniquely given by the complex ratio $\zeta=\xi^{1} / \xi^{0}$ of the probability amplitudes. Precisely this number $\zeta$ is the complex stereographic coordinate of the "good" quantization axis. 


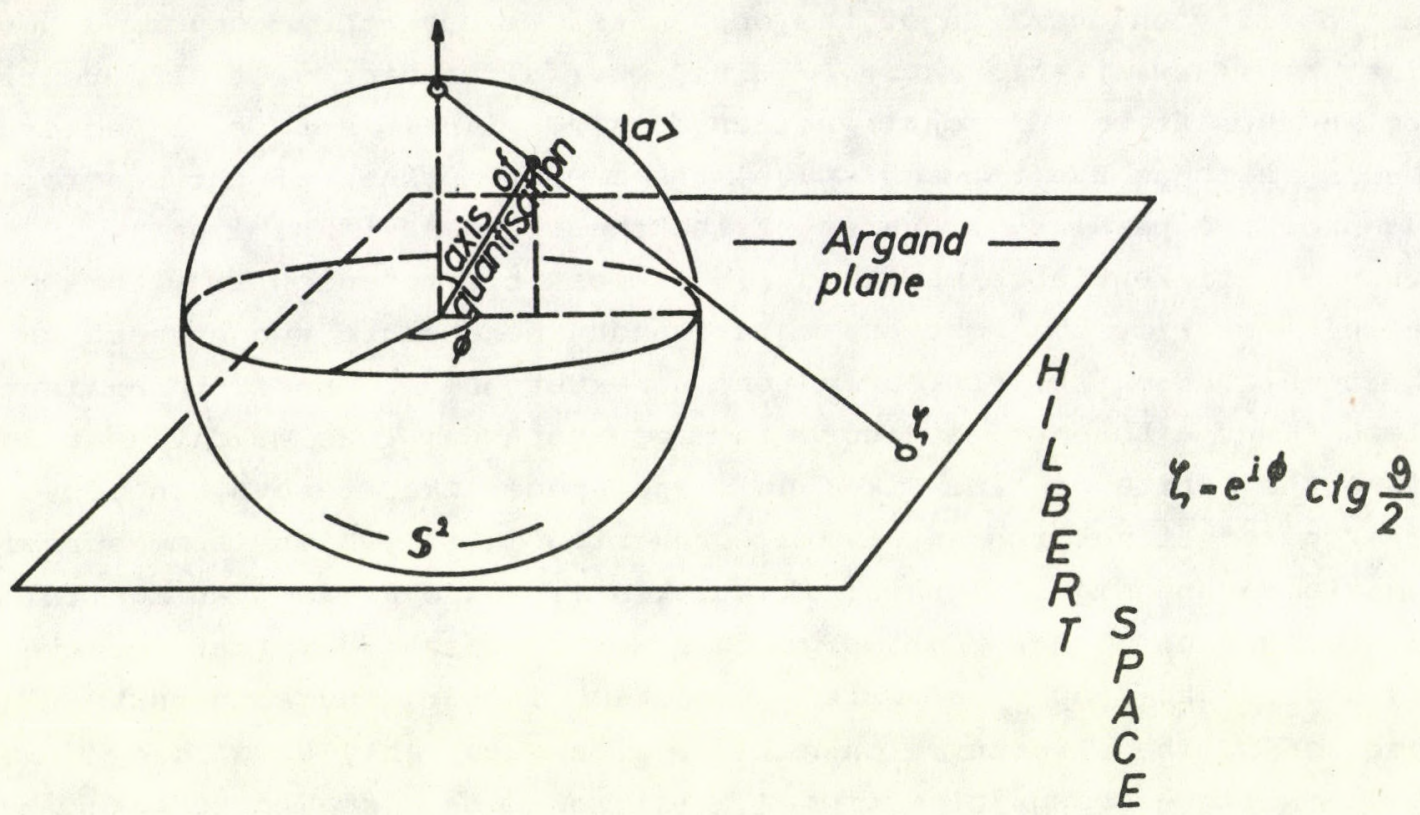

Fig. 2. The quantum states of a spin-1/2 particle

Twistor theory presents a relativistic version of the above picture. Here the two-sphere $S^{2}$ becomes the celestial sphere of some observer. Points of the sphere represent the past null directions of photons hitting the observer's eyes (Fig. 3). It is well-known by now that Lorentz transformations induce a conformal mapping of the sphere of null directions onto itself.

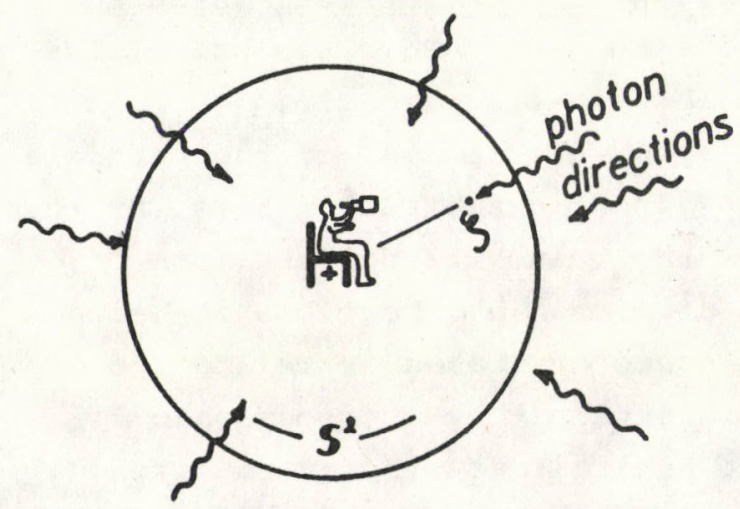

Fig. 3. The sphere 2 becomes in the relativistic picture the space of 4-momenta of photons hitting some observer's eye
In place of the non-relativistic isomorphism $O(3) \simeq \mathrm{SU}(2)$ we have $O(3,1) \simeq S L(2, \mathbb{C}) \simeq$ Möbius group.

The Möbius group is just the conformal group of $S^{2}$. In stereographic coordinates,

$\zeta^{\prime}=\frac{\alpha \zeta+\beta}{\gamma \zeta+\delta}, \quad \operatorname{det}\left[\begin{array}{ll}\alpha & \beta \\ \gamma & \delta\end{array}\right]=1$

When we enlarge $S^{2}$ to allow a full description of photons, we obtain twistor space.

There is a dual correspondence between points and lines in Minkowski space-time and in twistor space, respectively. In section 4 I shall show that this suggest naturally an extension os space-time into the complex. Less straightforward is the correspondence between fields describing states of 
zero-mass free particles and analytic twistor functions. This latter correspondence has been vigorously studied by the oxford research group, and resulted in the introduction of cohomological techniques in quantum field theory. Section 5 will explain how the positive frequency condition imposed on a zero-mass field leads to an interpretation of the twistor state function as a representative cocycle of a cohomology group element.

Equations of motion, when rewritten in twistor terms, exhibit a manifestly twistor invariant canonical structure. This is somewhat trivial for free particles since a twistor globally characterizes a free zero-mass particle. Penrose [7] has derived canonical twistor equations and covariant twistor quantization rules from a study of interacting zero-mass particles. Section 6 will present a method by which his results can be extended to massive particles $[8]$.

One of the most important unresolved problems of present day physics is the origin of rest-mass. Existing techniques such as the Higgs mechanism appear merely to shift the problem from one formulation to other. Not even twistor theory was able so far to yield a key to the origin of rest-mass. Nevertheless, it is possible to discuss massive particles and fields within the framework of twistor theory just as well as in other quantum field theories. Moreover, the twistor approach offers an insight into the problem by breaking the massive system into massless constituents. This gives rise to certain internal symmetries, which are useful in classifying quantum states $[4-20]$.

Although we are a long way from approaching the problem of rest-mass satisfactorily, I find it conceivable that the study of curved twistor spaces might bring us closer to this. Two or more massless particles chasing each other in a space curved by their presence can create mass out of no mass as perceived by a distant observer.

\section{WHAT ARE SPINORS GOOD FOR?}

One uses spinors of course to describe particles with spin. When parity is conserved, Dirac-spinors suffice. In more general situations one resorts to $\operatorname{SL}(2, \mathrm{C})$ spinors.

More profoundly, the utility of spinors depends on their close relation with zero-mass particles and with causality. Details follow.

In a flat space-time, given a vector $x^{a}$, we define the two-by-two matrix

$$
\left[x^{A A^{\prime}}\right]=\left[\begin{array}{cc}
x^{00^{\prime}} & x^{01^{\prime}} \\
x^{10^{\prime}} & x^{11^{\prime}}
\end{array}\right] \stackrel{\text { def }}{=} \frac{1}{\sqrt{2}}\left[\begin{array}{cc}
x^{0}+x^{1} & x^{2}+i x^{3} \\
x^{2}-1 x^{3} & x^{0}-x^{1}
\end{array}\right] \text {. }
$$

The action of a Lorentz transformation is then expressed 


$$
x^{A A^{\prime}}+\Lambda_{R^{A}}^{A} x^{R R^{\prime}} \bar{\Lambda}^{A^{\prime}} R^{\prime}
$$

where an overbar means complex conjugation. The matrix $\left[\Lambda_{R}^{A}\right]$ is unimodular, preserving the determinant

$$
2 \operatorname{det}\left[x^{A A}\right]=\left(x^{0}\right)^{2}-\left(x^{1}\right)^{2}-\left(x^{2}\right)^{2}-\left(x^{3}\right)^{2} .
$$

Take now a null vector, $l^{a}$, satisfying $\operatorname{det}\left[l^{A A^{\prime}}\right]=0$. The singular matrix $\left[l^{A A}\right]$ has the dyadic decomposition ensuring Hermiticity

$$
\ell^{A A^{\prime}}= \pm \xi^{A} \bar{\xi}^{A^{\prime}}
$$

Here the sign ambiguity is for adjusting the sign of $\ell^{\circ}$. The positive sign is chosen for $l^{a}$ future-pointing. Performing a Lorentz transformation (2.2) we find

$$
\xi^{\mathrm{A}}+\Lambda_{\mathrm{R}^{\mathrm{A}} \xi^{\mathrm{R}}} \quad \bar{\xi}^{\mathrm{A}^{\prime}}+\bar{\xi}^{\mathrm{R}^{\prime}} \bar{\Lambda}_{\mathrm{R}^{\prime}}^{\mathrm{A}^{\prime}} .
$$

Eqs. (2.4) and (2.5) reveal the close relationship between the null vector $\ell^{\mathrm{a}}$ and the rank-1 spinor $\xi^{\mathrm{A}}$.

From Eq. (2.3), we can write the metric, using the notation [9] $x^{a}=x^{A A^{\prime}}$,

$$
g_{a b}=\epsilon_{A B} \epsilon^{\prime} B^{\prime}
$$

where

$$
\left[\epsilon_{A B}\right]=\left[\epsilon_{A^{\prime}} B^{\prime}\right]=\left[\begin{array}{rr}
0 & 1 \\
-1 & 0
\end{array}\right]
$$

The quantity $\epsilon_{\mathrm{AB}}$ is the spinor metric, with its inverse defined by

$$
\epsilon^{\mathrm{CB}} \epsilon_{\mathrm{AB}}=\delta_{\mathrm{A}}^{\mathrm{C}} \text {. }
$$

The rules for raising and lowering spinor indices follow from transformation properties (2.5),

$$
\begin{gathered}
\xi_{A}=\xi^{R} \epsilon_{R^{\prime}} \\
\xi^{A}=\epsilon^{A B} \xi_{B}
\end{gathered}
$$

etc.

Skewing of three spinor indices gives zero since spinor indices can take only two distinct values. Hence, 


$$
\epsilon_{\mathrm{A}}\left[\mathrm{B} \epsilon_{\mathrm{CD}}\right]=0
$$

Transvecting with some spinor $n^{C D}$ we have

$$
n_{A B}-n_{B A}=\epsilon_{A B} n_{R}^{R}
$$

Thus, we can express the skew part of a spinor in terms of the trace.

Skew tensors similarly have nice properties in spinor notation. Take the Maxwell tensor, $\mathrm{F}_{\mathrm{ab}}=-\mathrm{F}_{\mathrm{ba}}$ :

$F_{A A^{\prime} B^{\prime}}=\frac{1}{2}\left(F_{A A^{\prime} B^{\prime}}-F_{B^{\prime} B^{\prime} A^{\prime}}\right)=\frac{1}{2}\left(F_{A A^{\prime}} B^{\prime}-F_{B^{\prime} A^{\prime} B^{\prime}}\right)+\frac{1}{2}\left(F_{B^{\prime}} A_{A B^{\prime}}-F_{B^{\prime} B^{\prime} A A^{\prime}}\right)$.

We have added and subtracted $\frac{1}{2} \mathrm{~F}_{\mathrm{BA}^{\prime}{ }^{\prime} \mathrm{AB}^{\prime}}$. Define the spinor

$$
\phi_{\mathrm{AB}}=\frac{1}{2} \mathrm{~F}_{\mathrm{AB}}{ }^{\mathrm{B}^{\prime}}
$$

Then, from skewness of $\mathrm{F}_{\mathrm{ab}}$ we find $\phi_{\mathrm{AB}}=\phi_{\mathrm{BA}}$. We next reshuffle the skewed indices in the parentheses of (2.12) using Eq. (2.11):

$$
F_{A^{\prime} B^{\prime} B^{\prime}}=\bar{\phi}_{A^{\prime} B^{\prime}} \epsilon_{A B}+\phi_{A B} \epsilon_{A^{\prime} B^{\prime}} \cdot
$$

We have expressed the Maxwell field tensor in terms of a symmetric spinor $\phi_{\mathrm{AB}}$. Defining the spinor derivative operator by

$$
\nabla_{\mathrm{a}}=\nabla_{\mathrm{AA}}
$$

we can put Maxwell's equations in the form

$$
\nabla^{\mathrm{AA}}{ }_{\phi_{\mathrm{AB}}}=0
$$

The field equations of the photon (a zero-mass particle) are thus suitably treated by using spinors.

\section{NULL TWISTORS}

Null twistors are represented by null lines in Minkowski space-time. Choose coordinates $x^{a}(a=0,1,2$ and 3$)$ with the metric $g_{a b}=\operatorname{diag}(1,-1,-1,-1)$, and origin 0 . Consider an arbitrary null line $\mathrm{L}$ with future-pointing tangent vector $\mathrm{p}^{\mathrm{a}}$ (Fig. 4). Let $\mathrm{x}^{\mathrm{a}}$ be some point of $\mathrm{L}$. We seek for coordinates characterizing $\mathrm{L}$. We can select, for example, the components of the tangent vector $\mathrm{p}^{\mathrm{a}}$ and of the momentum of $\mathrm{L}$ with respect to the origin,

$$
m^{a b}=x^{a} p^{b}-p^{a} x^{b}
$$




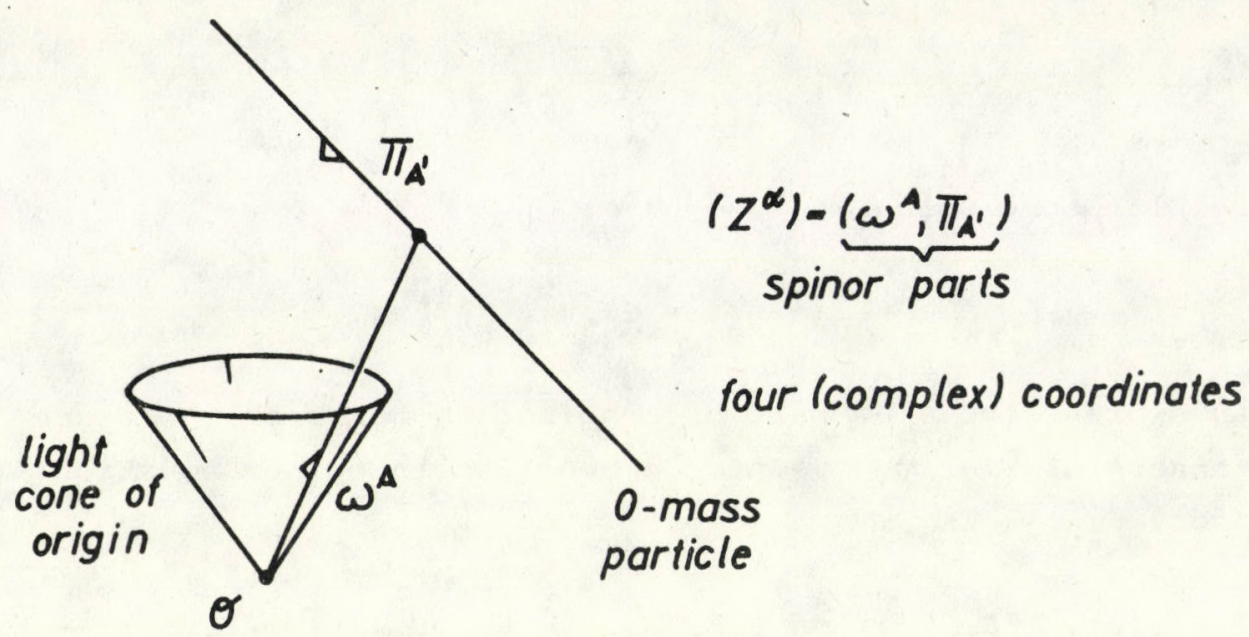

Fig. 4. The picture of a null twistor $z^{\alpha}$ in Minkowski space-time Here $\mathrm{m}^{\mathrm{ab}}$ is a skew tensor and $\mathrm{p}^{\mathrm{a}}$ a null vector. Hence we anticipate advantages in using a spinor description.

According to Eq. (2.4) we can set

$$
p^{a}=-\pi^{A} \pi^{A^{\prime}}
$$

Substituting in (3.1),

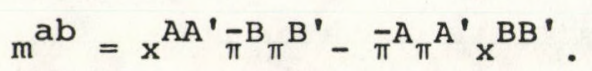

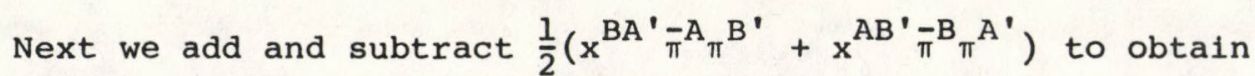

$\left.\left.m^{a b}=\left(\pi^{(A} x^{B}\right) A^{\prime} \pi^{B^{\prime}}-\pi^{(A} x^{B}\right) B^{\prime} \pi^{A^{\prime}}\right)+\left(\pi^{B} x^{A}\left(A^{\prime} \pi^{B^{\prime}}\right)-\pi^{A} x^{B}\left(A^{\prime} \pi^{B^{\prime}}\right)\right)$.

Here the indices in parentheses are symmetrized:

$$
\left.\bar{\pi}^{(A} x^{B}\right) A^{\prime} \stackrel{\text { def }}{\underline{1}} \frac{1}{2}\left(\bar{\pi}^{A} x^{B A^{\prime}}+\bar{\pi}^{B} x^{A A^{\prime}}\right) .
$$

The skew parts of (3.4) are rewritten, using (2.11)

$$
\mathrm{m}^{\mathrm{ab}}=1 \epsilon^{\mathrm{A}^{\prime} \mathrm{B}^{\prime}}{ }_{\omega}\left(\mathrm{A}-\bar{\pi}^{\mathrm{B}}\right)-1 \epsilon^{\mathrm{AB}-\left(\mathrm{A}^{\prime}{ }_{\pi} \mathrm{B}^{\prime}\right)}
$$

where

$$
\omega^{A}=-1 x^{A A^{\prime}} \pi_{A^{\prime}} \cdot
$$

Hence the spinors $\omega^{A}$ and $\pi_{A}$, together characterize the null line $L$. In particular, $\pi_{A}$, gives the direction of $L$ and $\omega^{A}$ the momentum with 
respect to the origin.

clearly, the spinor $\pi_{A}$, is independent of the choice of the point $x$ on L. When

$$
x^{A A^{\prime}} \rightarrow x^{A A^{\prime}}+a \pi^{-A} \pi^{\prime}
$$

we have

$$
\omega^{A} \rightarrow-i\left(x^{A A^{\prime}}+a \pi^{-A^{A} A^{\prime}}\right) \pi_{A^{\prime}}=\omega^{A}
$$

since $\pi^{A^{\prime}} \pi_{A^{\prime}}=0$.

Apart from singular cases we can choose the point $x$ to be the point of intersection of the line $L$ with the light cone of the origin. Then $x^{A A^{\prime}}$ has the form $x^{A A^{\prime}}=\xi^{A} \bar{\xi}^{A^{\prime}}$, and

$$
\omega^{A}=i\left(\bar{\xi}^{A^{\prime}} \pi_{A}{ }^{A}\right) \xi^{A}
$$

Inverting (3.10), we can write the vector $\mathrm{x}^{\mathrm{a}}$ as

$$
x^{A A^{\prime}}=\xi^{A} \bar{\xi}^{A^{\prime}}=\frac{1}{\left|\xi^{A} \bar{\pi}_{A}\right|^{2}} \omega^{A-A^{\prime}} .
$$

Hence we infer that the spinor $\omega^{A}$ lies in the direction of the point of intersection of $L$ with the light cone of the origin.

The pair

$$
\mathrm{z}^{\alpha}=\left(\omega^{\mathrm{A}}, \pi_{\mathrm{A}^{\prime}}\right) \quad \alpha=0,1,2,3
$$

is called a null twistor with spinor parts $\omega^{A}$ and $\pi_{A}$. The condition that $\mathrm{Z}^{\alpha}$ is null expresses that the line $\mathrm{L}$ is real. For, consider

$$
\omega^{A} \bar{\pi}_{A}=-i x^{A A^{\prime}}{ }_{A} \cdot \bar{\pi}_{A}
$$

Here the vector $\mathrm{x}^{\mathrm{a}}$ is real, so we can use the complex conjugate of Eq.

$(3.7)$ to write

$$
\omega^{A} \bar{\pi}_{A}=-\left(-1 x^{A} A_{A_{A}}\right) \pi_{A} ;=\bar{\omega}^{A^{\prime}} \pi_{A}
$$

Introducing the dual twistor $\overline{\mathrm{z}}_{\alpha}$ by

$$
\bar{z}_{\alpha}=\left(\bar{\pi}_{A^{\prime}}, \bar{\omega}^{\prime}\right)
$$

we neatly express the nullity condition (3.14) as the condition of vanishing twistor norm 


$$
z^{\alpha} \bar{z}_{\alpha}=\omega^{A} \bar{\pi}_{A}+\bar{\omega}^{\prime} \pi_{A^{\prime}}
$$

The effect of Lorentz transformations on twistors is obvious in the spinor notation. The Poincaré translations

$$
x^{a}+x^{a}+b^{a}
$$

leave the tangent spinor $\pi_{A}$, of $L$ unchanged and

$$
\omega^{A}+-i\left(x^{A A^{\prime}}+b^{A A^{\prime}}\right) \pi_{A^{\prime}}=\omega^{A}-1 b^{A A^{\prime}} \pi_{A^{\prime}} \cdot
$$

\section{SPACE-TIME GOES COMPLEX}

Consider two intersecting null lines in Minkowski space-time. The twistors describing each of the lines are

$$
z^{\alpha}=\left(\omega^{A}, \pi_{A},\right) \quad \text { and } \quad w^{\alpha}=\left(\tau^{A}, \rho_{A},\right)
$$

respectively. Selecting $\mathrm{x}^{\mathrm{a}}$ to be the point of intersection,

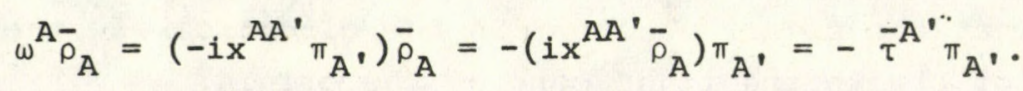

This is written in twistor form

$$
\mathrm{z}^{\alpha} \overline{\mathrm{w}}_{\alpha}=0
$$

In (4.3) we have the condition of incidence of the null lines corresponding to the twistors $z^{\alpha}$ and $w^{\alpha}$.

Observe now that a null line $L$ can be characterized by the set of all null lines incident with $L$. This set is given by all the solutions of Eq. (4.3) for $\overline{\mathrm{w}}_{\alpha}$. This set can be taken to define the twistor $z^{\alpha}$ where both $\mathrm{w}^{\alpha}$ and $z^{\alpha}$ are null. However, we can extend the validity of Eq. (4.3) to a nonnull $z^{\alpha}$. The non-null twistor $z^{\alpha}$ will be represented in Minkowski space-time by the congruence of null lines given by the solutions of (4.3) for $\overline{\mathrm{w}}_{\alpha}$. Depending on the sign of $z^{\alpha} \bar{z}_{\alpha}$, the congruence of null lines twists in a lefthanded or in a right-handed sense. (Hence is the term twistor).

A space-time point $x$ is fixed by two independent generators of the light cone of $x$ (Fig. 5). The corresponding two null twistors $w^{\alpha}$ and $z^{\alpha}$ satisfying $\overline{\mathrm{w}}_{\alpha} z^{\alpha}=0$ carry more information then just the coordinates $x^{a}$ : their $\pi$-parts define also a frame at $\mathrm{x}$. However, the components of their skewed product,

$$
x^{\alpha \beta}=z^{\alpha} w^{\beta}-w^{\alpha} z^{\beta}
$$




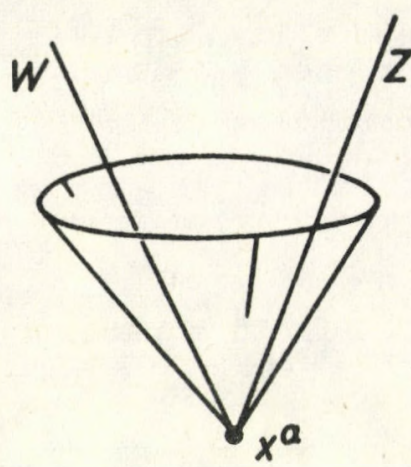

point $x^{\alpha} \Leftrightarrow x^{\alpha \beta}-w^{\alpha} z^{\beta}-z^{\alpha} w^{\beta}$

Fig. 5. Space-time point $x$ is fixed by two independent generators $W$ and $Z$ of its light cone

give just $\mathrm{x}^{a}$. The skew point twistor $\mathrm{x}^{\alpha \beta}$ has the conjugate $\overline{\mathrm{x}}_{\alpha \beta}$ and dual $x_{\alpha \beta}^{*}=\frac{1}{2} \epsilon_{\alpha \beta \gamma \delta} x^{\gamma \delta}$. For $x$ a real point, $\bar{x}_{\alpha \beta}=x_{\alpha \beta}^{*}$.

When one of the generators of the null cone moves out towards infinity, then we obtain a null plane in the 1imit. For a null line near infinity, the momentum with respect to the origin dominates the tangent vector of the line. At infinity we have

$$
z^{\alpha}=\left(\omega^{A} ; 0\right)
$$

The light cone at infinity is characterized by all 1 ts generators having the form (4.5). Thus it is described by the "infinity twistors"

$$
\left[I^{\alpha \beta}\right]=\left[\begin{array}{ll}
\epsilon^{A B} & 0 \\
0 & 0
\end{array}\right] \text { and } \quad\left[I_{\alpha \beta}\right]=\left[\begin{array}{ll}
0 & 0 \\
0 & \epsilon^{A^{\prime} B^{\prime}}
\end{array}\right] \text {. }
$$

The light cone at infinity remains what it is both under Lorentz transformations and under finite translations. The infinity twistors (4.6) are therefore Poincaré-invariant. They are not, however, preserved by inversions nor by dilations.

It is often useful in twistor theory to complexify space-time. When we allow $x^{a}$ to be complex, then the twistor equation (3.7) will have solutions for $\mathrm{x}^{\mathrm{a}}$ given an arbitrary $\mathrm{z}^{\alpha}$. The general solution has the form

$$
x^{A A^{\prime}}=x_{0}^{A A^{\prime}}+\xi^{A} \pi^{A^{\prime}}
$$

where $\xi^{\mathrm{A}}$ is arbitrary. Thus a twistor $\mathrm{z}^{\alpha}$ is represented in complex Minkowski space-time by a two-plane parametrized by $\xi^{\circ}$ and $\xi^{l}$. Any pair of points in this plane is connected by a null vector. The complexification of space-time emerging in a natural way in twistor theory appears to be a suitable vehicle for developing a quantum theory of geometry [7]. 


\section{FIELDS AND COHOMOLOGY}

One of the outstanding achievements of twistor theory is that is allows to generate the fields describing free zero-mass particles of spin

$$
s=0, \frac{1}{2}, 1, \frac{3}{2}, \ldots
$$

by contour integxals of analytic functions [10]. The field equations of a zero-mass, spin-s free particle are

$$
\nabla^{A A^{\prime}} \phi_{A^{\prime} B^{\prime} \ldots L^{\prime}}(x)=0
$$

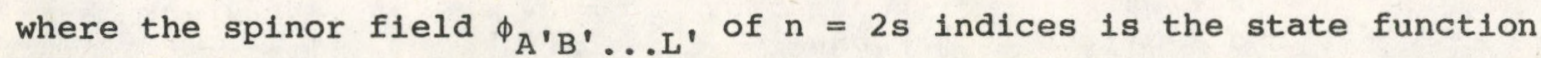
of the particle. Scalar fields $(s=0)$ are described by the wave equation

$$
\nabla_{\mathrm{AA}} \cdot \nabla^{\mathrm{AA}}{ }^{\prime} \phi(\mathrm{x})=0
$$

The field equations allow us to develop the field $\phi_{A^{\prime} B^{\prime}}$..... ' from its components specified on a 3-dimensional initial surface [13]. $\phi_{A^{\prime} B^{\prime} \ldots L^{\prime}}$ is, in effect a function of only 3 variables. Having quantization in mind, we consider these variables to be complex.

We define the three complex coordinates of projective twistor space $(\mathbb{P} T)$ through the ratios $\frac{z^{\alpha}}{z^{0}}\left(z^{0} \neq 0\right)$. Consider a homogeneous function $f\left(z^{\alpha}\right)$ of degree $-n-2$ :

$$
f\left(\lambda z^{\alpha}\right)=\lambda^{-n-2} f\left(z^{\alpha}\right)
$$

Let the spinor parts of $\mathrm{z}^{\alpha}$ be denoted

$$
\left(z^{\alpha}\right)=\left(-i x^{A A^{\prime}} z_{A}, z_{A}^{\prime}\right)
$$

The spinor function $\phi_{A^{\prime} B^{\prime} \ldots L^{\prime}}(x)$ of space-time coordinates is obtained by performing the contour integral

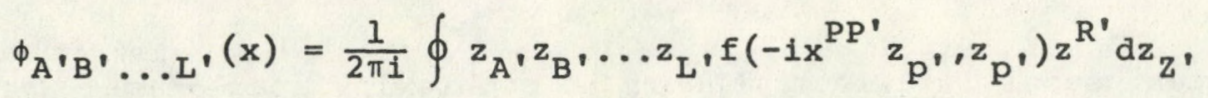

where the contour of integration avoids the singularities of $f$. It is a simple exercise to check that $\phi_{A^{\prime} B^{\prime} \ldots L^{\prime}}$ satisfies the zero-mass field equation (5.2). Conversely, it has been shewn by Bramson, Sparling and Penrose [11] that any sufficiently smooth solution of (5.2) can be expressed as a contour integral of the form (5.6).

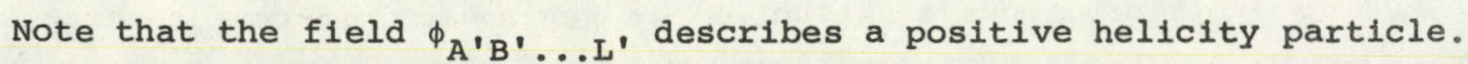
Hughston [12] has suggested that negative helicity states can be similarly generated from homogeneous twistor functions by the formula 


$$
\phi_{A B} \ldots L(x)=\frac{1}{2 \pi i} \oint \frac{\partial}{\partial \omega^{A}} \frac{\partial}{\partial \omega^{B}} \cdots \frac{\partial}{\partial \omega^{L}} f\left(\omega^{P}, z_{P^{\prime}}\right) z^{R^{\prime}} d z_{R^{\prime}} .
$$

Here the twistor function $f\left(z^{\alpha}\right)$ is again of homogeneity degree $-2 s-2$, the helicity $s$ being this time negative.

The singularities of the twistor function $f\left(z^{\alpha}\right)$ must be arranged so as to ensure the correct positive frequency behaviour of the field $\phi(x)$. In field theory it is customary to state positive frequency in terms of an $i \epsilon^{\prime}$ condition restricting the singularity structure in the process of analytic continuation. This $i \in$ condition is stated in twistor terms by requiring that the singularities of the twistor function lie in two disjoint sets of the domain $\mathbb{P} \mathbb{T}^{+}$of projective twistor space defined by $z^{\alpha} \bar{z}_{\alpha}>0$.

The integration variables $\pi_{0}$, and $\pi_{1}$, in (5.6) and (5.7) are homogeneous coordinates of the 1 -complex-dimensional projective space $C \mathbb{P}^{1}$. The $\mathrm{CP}^{1}$ is essentially the Riemann sphere as can be seen by introducing intrinsic coordinates for two overlapping regions in $C \mathbb{P}^{l}$, by $\zeta_{5}=\pi_{1}, / \pi_{0^{\prime}}$ (this excludes the point $\pi_{O^{\prime}}=0$ ) and by $n=\pi_{0} / \pi_{1}$, (excluding $\bar{\pi}_{1},=0$ ). The contour of integration is a closed curve in $\mathbb{C P}^{1}$ (Fig. 6). The contour can be deformed

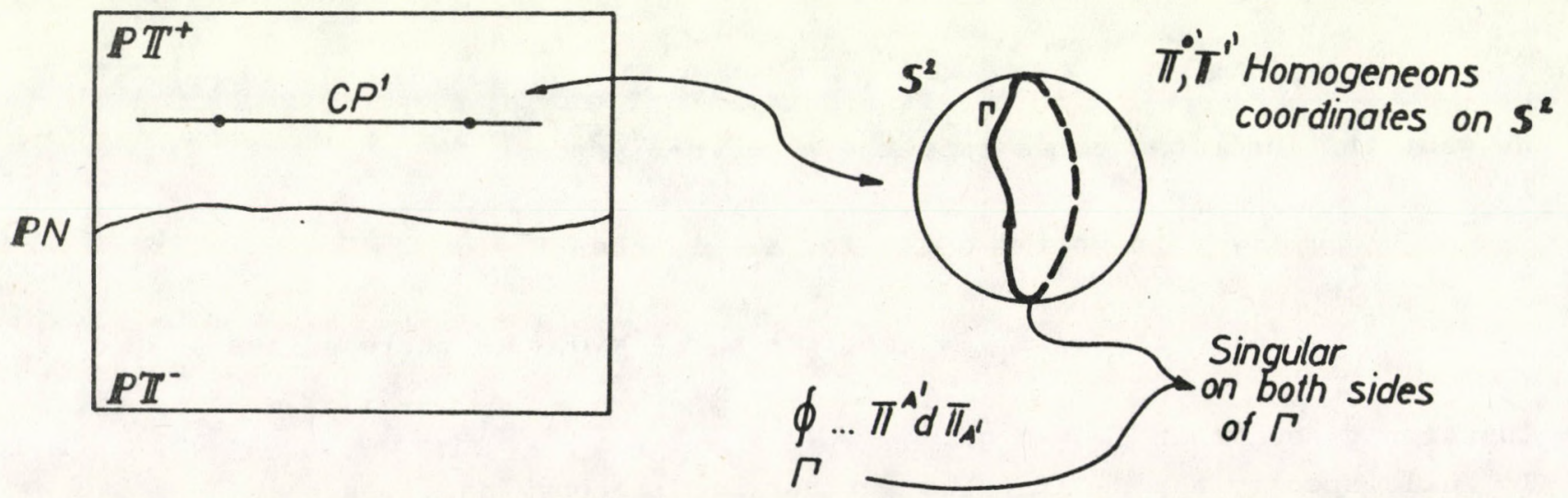

Fig. 6. The positive frequency condition

to a point unless singularities occur on both sides of the contour. We therefore choose the singularities of $f\left(z^{\alpha}\right)$ such that they fall in at least two disjoint sets of $\mathbb{P} \mathbb{T}^{+}$.

The contour integrals (5.6) and (5.7) vanish for integrands which can be split into two terms each regular on one side of the contour. Two twistor functions $f$ and $g$ are considered equivalent when

$$
f-g=h_{2}-h_{1}
$$

where $h_{1}$ is regular in some open set $U_{1}$ and $h_{2}$ is regular in the open set $U_{2}$ such that $\mathrm{U}_{1}$ and $\mathrm{U}_{2}$ together cover $\mathbb{C P}^{1}$. 
This leads us to sheaf cohomology theory.

Quite generally, consider a topological space $J$ which can be covered by a countable family of open sets $U_{i}(i=1,2, \ldots)$ : Consider then a collection of functions each defined on $p$-fold intersections of open sets $U_{i} \cap U_{j} \cap \ldots U_{\ell}$ and indexed

$$
f_{i j \ldots l}=f_{[i j \ldots l]} .
$$

(Note that the sign convention in (5.9) assigns an orientation with the intersections). The class from which the functions $f_{i j \ldots . . l}$ are chosen will be specified shortly. The collection of functions (5.9) is called a (p-1) cochain.

We next define the restriction map $\rho_{i}$ using a notation due to Hughston [12]. We write for a function $f_{j} \ldots l$ defined on $U_{j} \cap \ldots U_{l}$, when restricted to $U_{i} \cap\left(u_{j} \cap \ldots u_{\ell}\right)$,

$$
\rho_{1} f_{j} \ldots l \cdot
$$

The restriction map satisfies

$$
\rho_{i} \rho_{k}=\rho_{k} \rho_{i}
$$

We want the functions to satisfy the sheaf properties

$$
\begin{aligned}
& \text { (i) If } \rho_{i} f=\rho_{i} g \text { for all } i \text {, then } f=g . \\
& \text { (ii) If } \rho_{[i} f_{k]} \equiv \frac{1}{2}\left(\rho_{i} f_{k}-\rho_{k} f_{i}\right)=0 \text {, the there exists } a \text {. }
\end{aligned}
$$

function $g$ such that $f_{i}=\rho_{i} g$.

To fully specify the sheaf $\mathcal{\zeta}$, we further require our functions to be holomorphic or continuous etc.

The coboundary operator $\delta$ acting on $a(p-1)$ cochain $f=\left\{f_{j} \ldots \ell\right\}$ is defined by

$$
\left.f=\left\{\rho_{[i} f \ldots l\right]\right\}
$$

From (5.11) we have $\delta^{2} \mathrm{f}=0$.

The cochain $f$ is called a cocycle when $\delta f=0$. The cochain $f$ is a coboundary if $\mathrm{f}=\delta \mathrm{g}$ for some cochain $\mathrm{g}$. $\mathrm{p}$-cochains, $\mathrm{p}$-cocycles and p-coboundaries form each an Abelian group (addition of functions!)

A coboundary is also a cocycle since $\delta^{2}=0$. We can from the quotient group

$$
{ }^{\mathrm{p}}{ }_{\left\{U_{i}\right\}}(\mathcal{J}, \mathcal{J})
$$


the $\mathrm{p}^{\text {th }}$ cohomology group with coefficients in the sheaf $\Im$, with respect to the covering $\left\{U_{i}\right\}$. (The dependence on the covering is removed by considering only fine enough coverings).

Returning to our case $\mathcal{T}=\mathbb{C P}^{1}$, and $\mathcal{J}$ being the sheaf of analytic functions, it is sufficient to cover $\mathbb{P}^{1}$ by two open sets, $U_{1}$ and $U_{2}$. A single holomorphic function $\mathrm{f}_{12}$ already defines a $\mathrm{p}=1$ cochain by $\mathrm{f}_{12}=-\mathrm{f}_{21}$ and $\mathrm{f}_{11}=\mathrm{f}_{22}=0$. The holomorphic functions $\mathrm{f}_{12}$ and $\mathrm{g}_{12}$ belong to the same cohomology group element if they differ in a coboundary, $1 . e .$, if

$$
\left.f_{12}-g_{12}=\rho_{[1} h_{2}\right]
$$

where $h_{i}$ is holomorphic on $U_{i}$. This is precisely the condition (5.8) for the twistor functions providing the same space-time field.

Computations involving scalar products of twistor state functions require integration of many-variable functions over complicated contours. The corresponding procedure for Feynman graphs has been made fairly automatic. The introduction of cohomological machinery in twistor theory raises a hope for ending up again with a simple and effective set of calculations rules.

\section{INTERACTIONS AND QUANTIZATION}

A zero-mass particle moving freely in Minkowski space-time has a conserved four-momentum $\mathrm{p}_{\mathrm{a}}$ and anguilar momentum $\mathrm{m}_{\mathrm{ab}}$. In spinor notation,

$$
\begin{gathered}
p_{a}=\bar{\pi}_{A} \bar{\pi}_{A^{\prime}} \\
m_{a b}=\mu_{A B} \epsilon_{A^{\prime} B^{\prime}}+\bar{\mu}_{A^{\prime} B{ }_{A B}}
\end{gathered}
$$

where

$$
\mu_{A B}=i \omega\left(\bar{\pi}_{B}\right)
$$

Comparing with $(3.2)$ and $(3.6)$ we see that $\omega^{A}$ and $\pi_{A}$, are spinor parts of the twistor $\left(z^{\alpha}\right)=\left(\omega^{A}, \pi_{A},\right)$.

The quantities $\mathrm{p}_{\mathrm{a}}$ and $\mu_{\mathrm{AB}}$ are, by their transformation properties, again spinor parts of a symmetric twistor

$$
\left[A^{\alpha \beta}\right]=\left[\begin{array}{cc}
-2 i \mu^{A B} & p_{B^{\prime}}^{A} \\
p_{A}{ }^{B} & 0
\end{array}\right]
$$

We can rewrite this using the infinity twistor (4.6) and (6.2), in the twistor form 


$$
A^{\alpha \beta}=2 z^{(\alpha} I^{\beta) \gamma} \bar{z}_{\gamma} .
$$

The twistor $A^{\alpha \beta}$ is the kinematical twistor. It contains less information about the particle then does the twistor $z^{\alpha}$ since substitutions of the form.

$$
\hat{\mathrm{z}}^{\alpha}=\mathrm{e}^{i \vartheta} \mathrm{z}^{\alpha}
$$

preserve $A^{\alpha \beta}$. The description of the zero-mass particle by the kinematical twistor involves internal degrees of freedom constituting the group $\mathrm{U}(1)$. The twistor $z^{\alpha}$ facilitates a more fundamental description then does kinematical twistor. $z^{\alpha}$ may be considered as the square-root of the kinematical variables. Computing the helicity of the particle we find

$$
z^{\alpha} \bar{z}_{\alpha}=2 s
$$

Classically, the helicity scan take arbitrary real values.

The use of the kinematical twistor has the advantage that it can be extended to the description of massive particles. Naturally, we want to have a more primitive twistor description also for massive particles. We find a description by splitting the particle into zero-mass constituents. Such a splitting is familiar from the decomposition of a timelike. vector $\mathrm{p}^{\mathrm{a}}$ into the sum of null vectors $l_{1}^{a}$ and $l_{2}^{a}$. The decomposition is far from being unique since the plane in which the three vectors lie can be rotated about $\mathrm{p}^{\mathrm{a}}$ (Fig. 7). The kinematical twistor can likewise be decomposed,

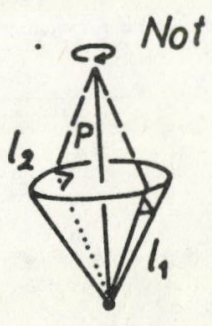

unique

$$
A^{\alpha \beta}=\sum_{i=1}^{n} A_{i}^{\alpha \beta} .
$$

$P^{\alpha}=l_{1}^{\alpha}+l_{2}^{a}$ Here $A_{i}^{\alpha \beta}$ are kinematical twistors describing the $i^{\text {th }}$

$$
\begin{aligned}
& P^{2}=m^{2} \\
& l_{1}^{2}=l_{2}^{2}=0
\end{aligned}
$$

Fig. 7. Decomposition of the timelike vector $p^{a}$ into the sum of two null vectors $\ell_{1}^{a}$ and $\ell_{2}^{a}$

zero-mass constituent. It is now possible to use a one-index twistor $z_{1}^{\alpha}$ for each of the constituents. We obtain a description of the massive particle by twistor $n$-plets, where $n \geq 2$.

The twistor $z_{i}^{\alpha}$ characterizes a free particle globalzy: it is a set of constants of the motion. This is why a Hamiltonian twistor approach to free particles would be somewhat trivial. However, twistor Hamiltonians are quite useful for describing interactions. The development of a Hamiltonian approach faces certain difficulties. These difficulties follow from our ignorance of the properties of curved twistor spaces. Investigations of interaction Hamiltonians have so far been restricted to situations in which a shock wave is sandwiched between two halves of flat Minkowski space-time. 
Penrose [7] has studied the scattering of a zero-mass particle on a weak plane-fronted gravitational shock wave described by the metric

$$
d s^{2}=2 d v(R d v+d u)-2 d \zeta d \bar{\zeta}
$$

where

$$
\mathrm{R}(\mathrm{v}, \zeta, \bar{\zeta})=\delta(\mathrm{v}) 2 \mathrm{Re} \mathrm{r}(\zeta)
$$

By integration of the equation of null geodesics he found that the twistor $\mathrm{z}^{\alpha}$ describing the particle changes by the infinitesimal amount

$$
\delta z^{\alpha}=-i \frac{\partial H}{\partial \bar{z}_{\alpha}}
$$

The Hamiltonian of this interaction has the form

$$
H=2 \operatorname{Re}\left\{\bar{z}_{\alpha} I^{\alpha \beta} \partial g\left(z^{\alpha}\right) / \partial z^{\beta}\right\}
$$

with the twistor function of the gravitational field (6.8)

$$
g\left(z^{\alpha}\right)=i\left(z^{2}\right)^{2} \int_{x_{0}}^{-i z^{1} / z^{2}} \bar{r}(x) d x .
$$

The twistor quantization rules can be inferred from the role the twistor variables $\mathrm{z}^{\alpha}$ and $\overline{\mathrm{z}}_{\alpha}$ play in the canonical equations (6.9):

$$
\left[z^{\alpha}, z^{\beta}\right]=0, \quad\left[z^{\alpha}, \bar{z}_{\beta}\right]=\delta_{\beta}^{\alpha}
$$

These quantum commutators are then consistent with the commutation properties of the generators $\mathrm{p}^{\mathrm{a}}$ and $\mathrm{m}^{\mathrm{ab}}$ of the Poincaré group. The corresponding quantum rules for twistor constituents of massive particles have been investigated by Tod and Perjés [8]. From the canonical structure of the twistor equations of massive particle scattering they find

$$
\left[\mathrm{z}_{i}^{\alpha}, \mathrm{z}_{\mathrm{k}}^{\beta}\right]=0, \quad\left[\mathrm{z}_{i}^{\alpha}, \overline{\mathrm{z}}_{\beta}^{\mathrm{k}}\right]=\delta_{\beta}^{\alpha} \delta_{i}^{\mathrm{k}}
$$

M. Ginsberg has subsequently derived the anti-commutation properties of fermion matrices from the twistor quantization rules.

The decomposition of the kinematical twistor into zero-mass terms introduces internal symmetries into the twistor description [18] (Cf. Table). The kinematical twistor is invariant under the transformations of the constituent $n$-twistors

$$
z_{i}^{\alpha} \rightarrow U_{i}^{j}\left(z_{j}^{\alpha}+I^{\alpha \beta} \Lambda_{j k} \bar{z}_{\beta}^{k}\right)
$$


Table 1.

\begin{tabular}{|c|c|c|c|}
\hline $\begin{array}{l}\text { No. of twistor } \\
\text { constituents }\end{array}$ & $\begin{array}{l}\text { No. of internal } \\
\text { parameters }\end{array}$ & $\begin{array}{l}\text { No. of algebraic } \\
\text { constraints }\end{array}$ & Group Interpretation \\
\hline 1 & 1 & 0 & U(1) $\begin{array}{l}\text { zero-mass } \\
\text { particles }\end{array}$ \\
2 & 6 & 0 & O(3)冈E(2) leptons \\
4 & 14 & 1 & ISU(3) noncharm \\
& 22 & 6 & $?$ \\
\hline
\end{tabular}

Table of twistor internal symmetry groups

where $\left[U_{i}^{j}\right]$ is an nxn unitary matrix and $\left[\Lambda_{j k}\right]$ is skew complex matrix. When $n \geq 3$, certain algebraic constraints on the internal twistor groups (6.14) follow from the 4-dimensional nature of twistor space.

For a 3-twistor particle there is a single algebraic constraint on the internal group which can be eliminated by requiring the [U $\left.U_{i}^{j}\right]$ be an $\mathrm{SU}(3)$ matrix. In this way I obtained [15] the 14-parameter ISU(3) group, which appears naturally as the group of isometries of the 3-complex dimensional unitary space, with metric $d z^{i} d \bar{z}_{1}$. It is then possible to describe the intrinsic properties of the particle by state functions defined on this internal unitary space. Investigation of the algebraic constraints on a 4-twistor particle shows [16] that it can also be described by structures in the unitary 3-space: this time we have two points rather than one with oppositely pointing (unitary) spins.

3- and 4-twistor particles are extended objects in space-time whereas 2-twistor systems are pointlike. The suggestion is that leptons can be described as two-twistor systems and hadrons as more complicated objects. The issue is not settled, however [12]. We can be guided by the observation [21] that the 2-twistor systems possess the internal twistor group $O(3) \otimes E(2)$ which is not much larger than (and in fact contains) the weak interaction symmetry group SU(2)®U(1) of the Weinberg-Salam theory.

It is tempting to classify known particles into representations of the twistor symmetry groups. Various lepton schemes have been proposed by Sparling and Hughston [12]. The discrete representations of the 14-parameter ISU(3) group have been worked out by Perjés and sparling [19]. 


\section{ACKNOWLEDGMENT}

I am most grateful to Dr. L.P. Hughston for patiently guiding me to basic notions of cohomology theory.

\section{REFERENCES}

[1] R. J. Eden, P. V. Landshoff, D. I. Olive and J. C. Polkinghorne: The Analytic S-Matrix (Cambridge University Press, 1966)

[2] R. Penrose: Techniques of Differential Topology in Relativity, Regional Conference Series in Applied Mathematics, Vol. 7 (SIAM, 1972)

[3] K. Symanzik: Casimir Effect and the Schrödinger Representation of Renormalizable Quantum Field Theory, this Volume.

[4] R. Penrose: The Geometry of the Universe, in Mathematics Today, Ed. L. A. Steen (Springer, 1978)

[5] R. Geroch: Spacetime Structure from a Global Viewpoint, in General Relativity and Cosmology, proceedings of the course 47 of International School of Physics "Enrico Fermi" (Academic, 1971)

[6] R. Penrose: A Brief Outline of Twistor Theory. University of Oxford preprint, 1980

[7] R. Penrose, Int. J. Theor. Phys. 1,61 (1968)

[8] K. P. Tod and Z. Perjés, GRG Journal ㄱ, 903 (1976)

[9] R. Penrose: The Structure of Space-Time, in Battelle Rencontres, Eds. C. M. DeWitt and J. A. Wheeler (Benjamin, 1967)

[10] R. Penrose, J. Math. Phys. 10, 38 (1969)

[11] R. Penrose: Twistor Theory: Its Aims and Achievements, in Quantum Gravity: An Oxford Symposium, Eds. C.J. Isham, R. Penrose and D. W. Sciama (Clarendon, 1975)

[12] L. P. Hughston: Twistors and Particles, Lecture Notes in Physics, Vol. 97. (Springer, 1979)

[13] R. Penrose: Quantization of Genérally Covariant Field Theories (preprint, 1963), E. T. Newman and R. Penrose, Proc. Roy. Soc. A305, 175 (1968)

[14] R. Penrose: Twistors and Particles, in Quantum Theory and the Structure of Time and Space, Eds. L. Castell, M. Drieschner and C. F. von Weizsäcker (Carl Hanser, 1975)

[15] z. Perjés, Phys. Rev. Dll, 2031 (1975)

[16] z. Perjés, Phys. Rev. D20, 1857 (1979)

[17] z. Perjés, Reports Math. Phys. 12, 193 (1977) 
[18] R. Penrose, Reps. Math. Phys. 12, 65 (1977)

[19] z. Perjés and G. A. J. Sparling: Twistor Structure of Hadrons, in Advances in Twiotor Theory, Eds. L. P. Hughston and R. Ward (P1tman, 1979)

[20] G. A. J、 Sparling, University of P1ttsburgh preprint, 1980.

[21] A. S. Popovich: Twistor Classification of Elementary Particles. Oxford, 1978, M. Sc. Thesis. 




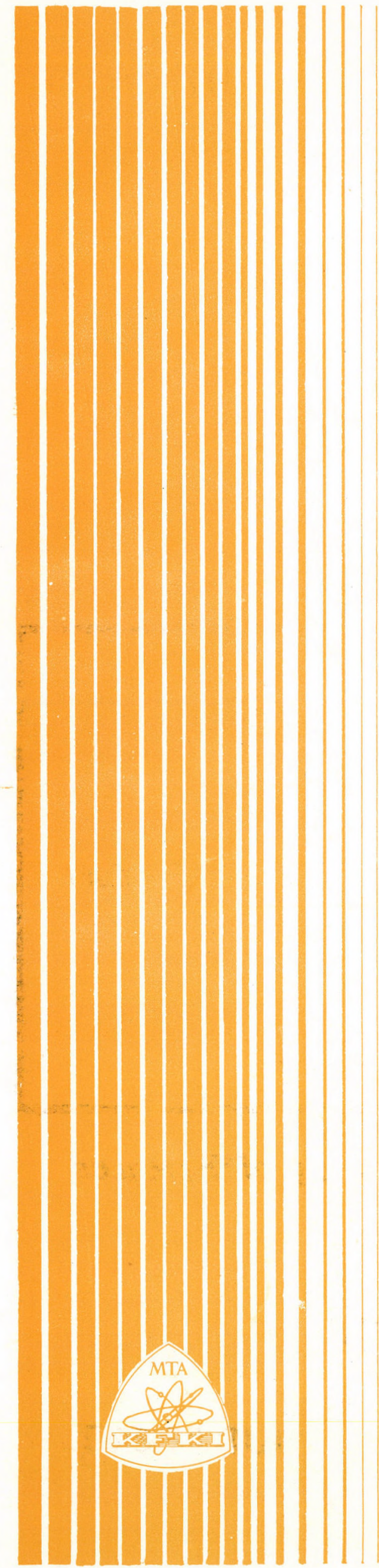

Kiądja a Központı Fizika1 Kutató Intézet Felelỗs kiadó: szegô károly. Szakmal lektor: Lukács Béla Nyelvi lektor: Sebestyén Akos Példányszám: 370 Törzsszám: 80-603 Készült a KFKI sokszorositó Uuzemében Budapest, 1980. október hó 